\title{
The Tevatron Ionization Profile Monitors
}

\author{
A. Jansson, T. Fitzpatrick, K. Bowie, R. Kwarciany, C. Lundberg, \\ D. Slimmer, L. Valerio, J. Zagel
}

Fermilab, Batavia, Illinois

\begin{abstract}
In designing an ionization profile monitor system for the Tevatron some novel approaches were taken, in particular for the readout electronics. This was motivated by the desire to resolve the individual bunches in both beams simultaneously. For this purpose, custom made electronics originally developed for Particle Physics experiments was used to provide a fast charge integration with very low noise. The various parts of the read-out electronics have been borrowed or adapted from the KTev, CMS, MINOS and BTev experiments. The detector itself also had to be modified to provide clean signals with sufficient bandwidth. The system design will be described along with the initial results.
\end{abstract}

Keywords: Enter Keywords here.

PACS: 07.05.Hd 07.77.Ka 29.27.-a

\section{INTRODUCTION}

As part of the Run II machine upgrades, an ionization profile monitor (IPM) has been developed for the Tevatron. The aim was to be able to measure beam size oscillations during proton and pbar injection, as well as to study beam size evolution during ramp and squeeze. Compared to other machines where IPMs have been installed, at Fermilab and elsewhere, one obvious difference is the dual beams. The Tevatron collider has both a proton beam and a counter-rotating pbar beam circulating on separate helical orbits. To cover both beams at injection, a relatively wide active area is required. On the other hand, to resolve the beam size at top energy, the granularity must be quite fine, leading to a large number of channels.

The second issue related to the dual beams is how to distinguish between them. Although the spatial separation provides some means of doing this, there were several reasons not to rely on this alone. The helix separation changes during the ramp and squeeze, and the projected separation in any given plane is not necessarily adequate to avoid overlap of the two beam profiles. Furthermore, the helix has been subject to several revisions, making it uncertain to rely on the spatial separation at a given point in the machine to tell the two beams apart.

Therefore, it was decided to separate the two beams by time of arrival, which requires single bunch resolution. This also allows freshly injected bunches to be studied separately from bunches already circulating in the machine. To obtain such a time resolution, the detector must not only have a short time constant, but also be free from resonances and any spurious signals (such as electromagnetic interference created by the beam passage) over a large bandwidth. 
The high charge concentration in the Tevatron beam makes it imperative to collect electrons rather than ions, so that a magnetic field can be used for focussing. Ions would also have been too slow moving to allow single bunch resolution.

Another challenge in the Tevatron is the low vacuum pressure required for collider operation. In order to obtain enough ionization electrons to reconstruct the width of a single bunch, the vacuum had to be locally increased by two orders of magnitude.

\section{INSTRUMENT DESIGN}

\section{Detector}

Ionization electrons are accelerated in a sweep field of $\sim 115 \mathrm{kV} / \mathrm{m}$, and focused by a parallel magnetic field of $0.2 \mathrm{~T}$. At ground potential, they pass thru a wire mesh, and are then slightly decelerated before they hit a large micro-channel plate (MCP). The MCP amplifies the incoming electron signal and the result is detected on anode strips. There are 200 copper strips on a ceramic board, each $10 \mathrm{~cm}$ long with a $0.25 \mathrm{~mm}$ pitch, giving a total active area $5 \mathrm{~cm}$ wide. The strips are connected via $50 \Omega$ resistors to flexible circuit boards, which carry the signals to the five DB50 feed-throughs that bring the signals out of the vacuum. To suppress any beam-induced parasitic signals due to electromagnetic coupling, the MCP, anode board, and signal wires are enclosed in a Faraday cage, of which the grounded wire mesh above the MCP forms an integral part.

The sweep field is generated by a wire grid at $-10 \mathrm{kV}$ potential, with a solid high voltage plate behind it at a slightly lower voltage. Ions that are accelerated in this direction pass thru the grid and strike the high voltage plate, but any secondary electrons generated in the process are inhibited by the grid voltage and consequently will not pass thru to be accelerated towards the MCP.

The electrons generated by the beam are generally collected within a few nanoseconds. However, at top energy, with a beam size of about $0.5 \mathrm{~mm}$, some of the electrons may temporarily be trapped in the potential well created by the positively charged proton bunch. Due to the magnetic field, this mainly generates a distortion in the time structure of the electron signal, and does not significantly affect the transverse distribution of the detected electrons.

Ionization electrons are generated at an average potential energy of $5 \mathrm{kV}$, and have about $3-5 \mathrm{keV}$ of kinetic energy when they hit the MCP. This is roughly the energy where the MCP has its highest detection efficiency. Since generally less than 1000 ionization electrons are expected per bunch, it is necessary to run at a high MCP gain, but with 36 proton bunches in the machine, the average signal current draw in the center of the beam profile start saturating the MCP at gains above 10000. This is about the highest gain that be obtained with a single MCP. Dual MCPs could be used, but each plate would then have to be operated at a very low gain causing higher noise. 
Due to the lower bias voltage, the second plate (which still would have to produce the same output current) would also saturate at an even lower total gain. Therefore, it was decided to use a single extra-high bias current MCP.

The detector is also equipped with a electron generator plate (EGP) embedded in the high voltage plate. The voltage across this plate is normally zero, but it is possible to rearrange the voltages of the high voltage plate, screen and EGP to emit elctrons in the direction of the MCP. This feature will be used to track changes in the MCP gain.

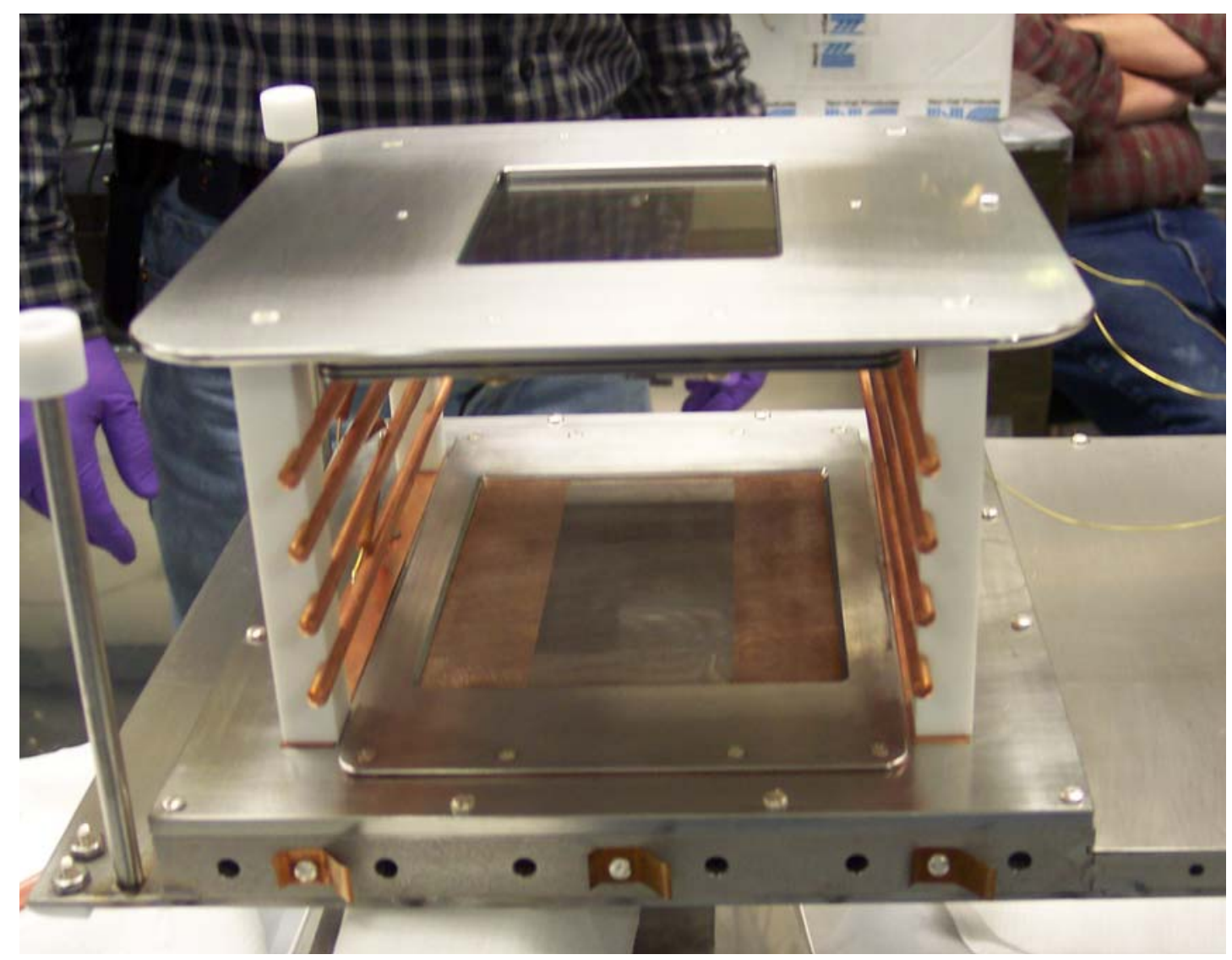

FIGURE 1. The detector of the Tevatron IPM. It is mounted to a large vaccum flange for easy installation.

\section{Overall layout}

The active part of the detector inserts into a chamber inside an electromagnet that provides the B-field. The chamber is mounted on motorized stands to allow the detector anode strips to be accurately aligned with the beam. The stands also permit the active area of the detector to be moved with respect to the beam position. This proved very helpful for the initial testing when the active area was limited, and may later be used to study e.g. channel-to-channel gain variations by scanning the detector position with respect to the beam. 
Electromagnets were chosen over permanent magnets, so that the magnetic field could be reduced or turned off. This feature was also very useful for initial testing.

Each detector, horizontal and vertical, has a compensation magnet to cancel the effect on the beam orbit. The residual orbit distortion from this two-magnet bump was estimated to be less than $0.1 \mathrm{~mm}$, and no measurable distortion could be detected by the beam position monitors when the magnetic was turned on and off.

The magnets, stands, and vacuum chambers were installed during the 2004 long shutdown, in preparation for the installation of the detector units.

\section{Vacuum}

The vacuum in the Tevatron warm straight section is generally in the high $10^{-10}$ Torr to low $10^{-9}$ range. In order to make a reasonable single bunch measurement, a pressure in the mid $10^{-8}$ range is needed. For a nominal proton bunch at injection, this will produce about 1000 electron-ion pairs in the $10 \mathrm{~cm}$ active length of the detector, and about 300 pairs for a pbar bunch (due to the lower intensity).

To locally increase the pressure, a calibrated nitrogen leak is used. In order to avoid excessive pressure transients when the system is turned on and off, the nitrogen source is located on a separate chamber, which is actively pumped to keep the pressure in the high $10^{-8}$ Torr range. By opening a gate valve, nitrogen is allowed to flow thru a small orifice to the main Tevatron vacuum system, midway between the two detectors. The pressure increase is localized by differential pumping on both sides of the IPMs.

\section{DATA ACQUISITION SYSTEM}

\section{Conceptual design}

It was clear from the beginning that in order to measure single bunches in the Tevatron, one would need sensitivity in the femto-Coulomb range and a time resolution (integration time) of less than 100ns. This kind of resolution is regularly obtained in Particle Physics experiments, which is why a solution to the DAQ problem was sought there.

After investigating several available options, it was found that the most suitable one was the CMS version of the Fermilab-designed QIE (charge integrator encoder) chip[1]. This chip utilizes parallel integration circuitry to obtain continuous back-toback integration intervals at frequencies between 7 and $53 \mathrm{MHz}$ with essentially no deadtime. It also includes a digitizer with an LSB of $2.6 \mathrm{fC}$ and a dynamic range of $>1 \mathrm{e} 4$ in logarithmic mode, or $0.9 \mathrm{fC}$ and a much smaller dynamic range in linear mode. The result is output as a 5 bit pseudo-logarithmic mantissa, and a 2 bit exponent (range setting). A 2 bit CAP ID value is also output, indicating which of the four integrator circuits was used.

The clock frequency was chosen to be $2 / 7$ of the RF, which is about $15 \mathrm{MHz}$. Using a multiple of $\mathrm{RF} / 7$ is required to stay synchronous with bunches in all three trains without running at the full RF frequency. The second harmonic was chosen to allow 
positive separation between protons and pbar, which are typically separated by around $100-120 \mathrm{~ns}$ at the location of the IPMs, depending on the cogging state.

Although each detector has 200 channels, only 128 are instrumented. Of the five DB50 vacuum feed-throughs, each carrying 40 channels, three are cabled to instrument all 40 anode strips, whereas the other two are cabled to instrument only every tenth strip. By swapping DB50 connectors on the detector, the densely instrumented area can then easily be moved, if needed.

\section{Timing card}

The timing card, which is implemented as a standard PCI card, listens to the Tevatron Beam Sync Clock (TVBS), which is an encoded timing signal with a 1/7 RF carrier frequency locked to the Tevatron RF. The board derives the front end clock $(2 / 7 \mathrm{RF})$ from the carrier, and decodes and transmits proton injection and data acquisition trigger events. The board also listens to the Antiproton Beam Sync Clock (APTVBS) for the pbar revolution marker and pbar injection event.

The timing signals are distributed on regular Cat-5 cables. The four differential pairs are used as follows:

1. 2/7 RF front end clock

2. Proton revolution marker

3. Pbar revolution marker

4. Encoded trigger/control signal

The encoded signal contains injection events, trigger events, and control commands to reset the board and change the QIE mode. In the tunnel, the signals are buffered by a fanout card, and distributed to the 16 integrator/digitizer cards used per IPM system.

Logic functions for the timing card are implemented in an FPGA. A separate version of the same card, with a different firmware, decodes general timing events (TCLK) and machine data (MDAT). This information is made available directly to the analysis software, and is not distributed to the front-end cards.

\section{Front-end integrator/digitizer card}

The front-end integrator/digitizer card was based on a prototype built for the KTev experiment. It has eight analog input channels, each with an associated reference signal. These are fed through two custom D-sub coax connectors housing 4 signalreference pairs each. Each signal-reference pair is then connected to a QIE chip. The pedestal of each QIE integrator can be individually set using DIP switches.

Timing information and ADC clock is provided thru an RJ45 connector. The frontend card maintains a number of cyclical counters, based on this information, namely

1. Proton sample number (clock cycles since proton revolution marker)

2. Pbar sample number (clock cycles since pbar revolution marker)

3. Turn number (number of proton revolution markers since last trigger)

The output data from the 8 QIEs is combined with timing and counter information, serialized and sent on a 1.6Gbps optical link. The serializer chip[2] used for this was developed by CERN, also for the CMS experiment, and is radiation hard. For stability, 
it is driven by a local oscillator, rather than locked to the front end clock. The logic functions of the card are implemented in an FPGA. One-time programmable antifuse FPGAs were used to be radiation insensitive. The cards have been tested using a charge-injected injection system based on a laser-PMT setup. The achieved rms noise level is about $1.8 \mathrm{fC}$ per sample at $15 \mathrm{MHz}[3]$.

\section{Data buffer card}

The data buffer card can handle eight incoming optical links (64 channels). It sorts the data and places it in buffer memory. There is $512 \mathrm{MB}$ of memory, enough for continuous acquisition of 20000 turns. Since only 72 out of 318 samples generally contain interesting data, the board can also be set up to sparsify the data on-the-fly, by saving only data with specific sample counter values. In this way, 90000 turns can be acquired for $36+36$ bunches, and 2 million turns for a single bunch.

The buffer board also does basic consistency checking. It maintains the same counters as the front-end boards, and verifies that all incoming links have synchronized counter values. It also verifies that all the QIEs are in the correct mode, and have synchronized CAP IDs.

Data can be saved in two formats. The standard format has all timing information except the counters stripped from the data, and all QIE values are aligned to 8bit boundaries for efficiency. There is also a built-in lookup table for conversion from native QIE quasi-exponential numbers to 8 bit linear values. The debugging format maintains all the timing information for a subset of the links in a more compressed format.

The board, which doubled as a prototype of the Level 1 trigger data buffer for the BTev experiment, uses a large FPGA with built-in Gbps de-serializers, and is implemented as a PCI64 card. The wider PCI bus was chosen to increase the data transfer rate, but due to internal limitations in the PCI controller chip the advantage of this has so far been limited.

\section{Software}

The front-end software is written in LabView and runs on a server PC with dual Xeon processors. A server motherboard was used since the PCI64 bus is not commonly available in regular PC motherboards. The base drivers for the PCI cards were generated using Jungo's WinDriver and PLX Technologies PLX PCI SDK, and were customized to exploit each cards unique capability.

\section{BEAM MEASUREMENTS}

\section{Initial Test Setup}

The original plan was to install the detectors during the autumn 2005 shutdown. This shutdown was later postponed to the spring of 2006, but a magnet failure in 
December caused unforeseen downtime, allowing the installation of one of the detectors.

For initial testing, a reduced DAQ system consisting of 5 front-end cards connected to a single buffer board was used. This setup provides 40 channels, which corresponds to $10 \mathrm{~mm}$ of active width, enough to study one beam at the time.

Due to a problem with sparking and vacuum degradation when running with full fields, the initial measurements were typically made with $25 \%$ magnetic field (500 Gauss) and $75 \%$ electric field $(7.5 \mathrm{kV})$.

The nitrogen leak system, which had been previously successfully tested, was not used for the initial detector testing, since a vaccum problem caused the pressure in the area to be sufficient for IPM measurements.

Both the sparking problem and the vacuum problem were later understood and corrected during the shutdown.

\section{First results}

Initial data taking was mainly done parasitically during stores or while injecting. Bunch profiles obtained during stores (see Figure 2) yielded beam sizes in the range expected from flying wire measurements, after correcting for the reduced magnetic field. Turn-by-turn analysis of single bunches during stores indicate a beam size sensitivity of about $5 \%$.
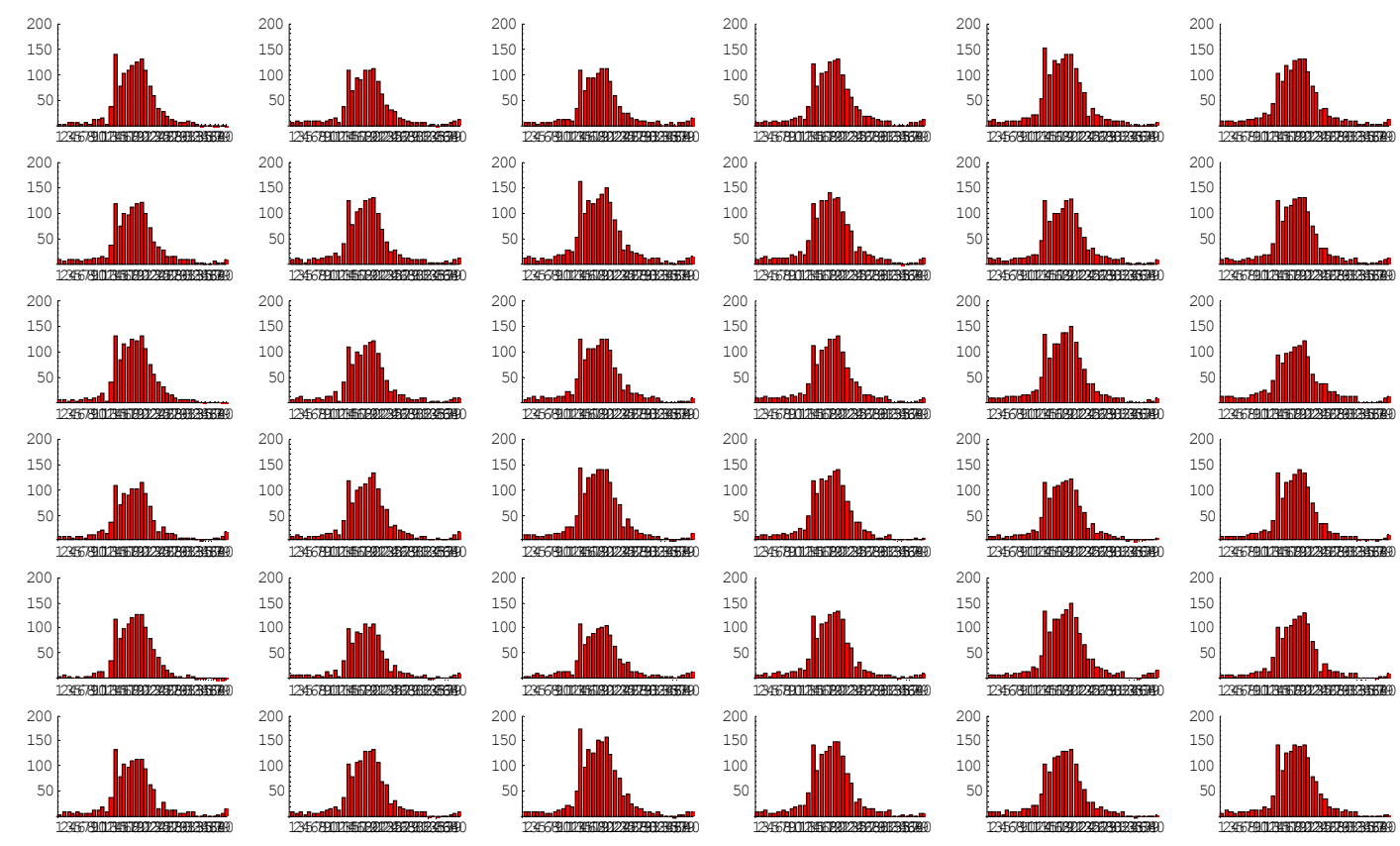

FIGURE 2. Single pass profiles for all 36 proton bunches at $980 \mathrm{GeV}$, measured with $25 \%$ B-field. Measured profile widths are in the range $0.7-0.9 \mathrm{~mm}$, which corresponds to real beam sizes of $0.5-0.75$ $\mathrm{mm}$ after correction for the lower magnetic field. This is in good agreement with expectation, although detailed comparative measurements have not yet been made. 
Measurements of turn-by-turn beam size at injection indicated a relatively large oscillation (see Figure 3). The frequency of the beam-size oscillation was found to be twice the betatron frequency, as expected. Due to lack of time, correction of the injection matching to reduce the oscillation amplitude was not attempted.

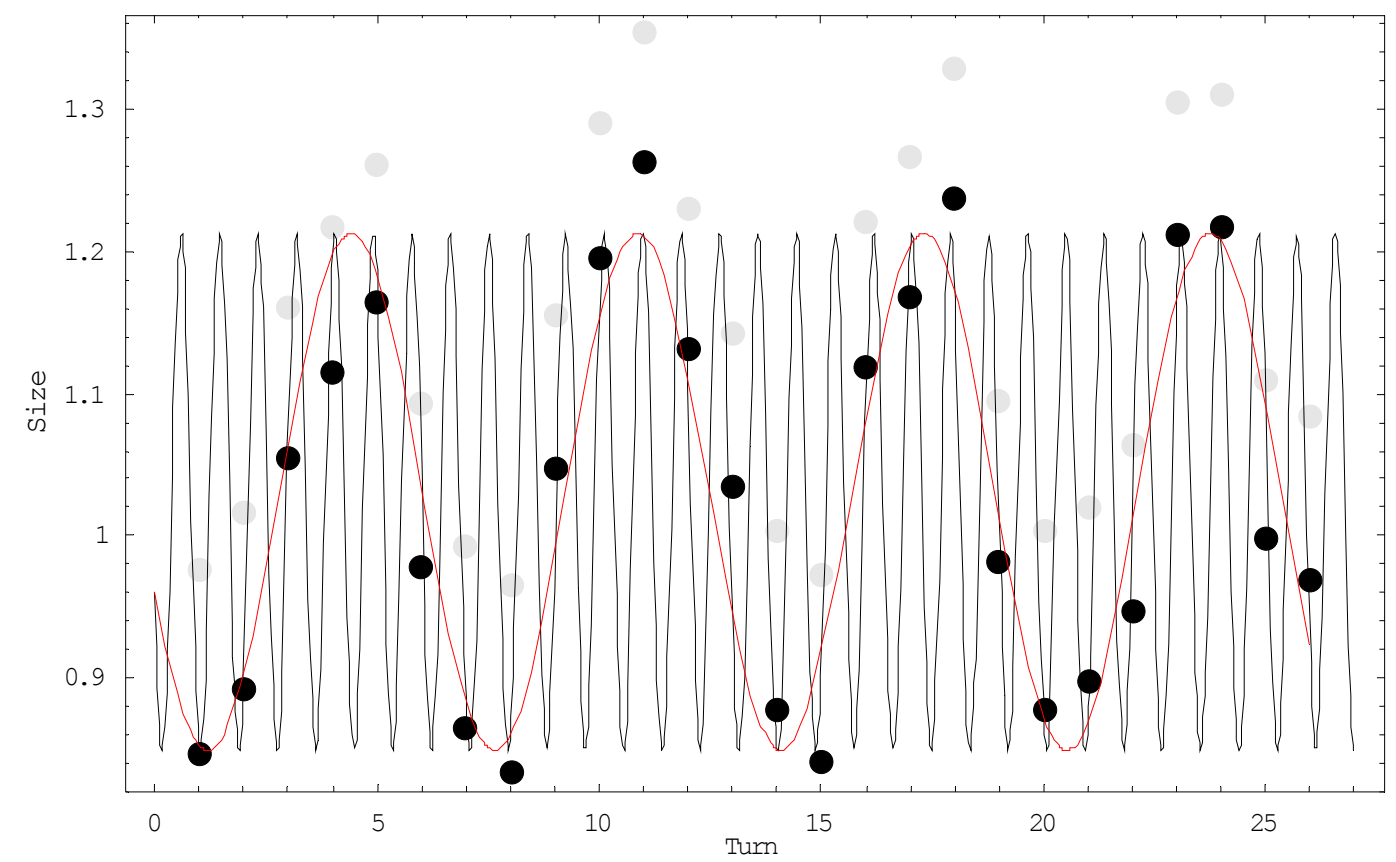

FIGURE 3. Turn-by-turn single-bunch measurement at injection with $25 \%$ magnetic field. The grey dots are the measured rms profile widths, the black dots the inferred bunch sizes (taking into account the reduced magnetic field), and the solid lines are the fits to sine functions at the quadrupole frequency $2 \mathrm{Qv}$, and its aliased frequency $\bmod (2 \mathrm{Qv})$.

\section{SUMMARY AND OUTLOOK}

The initial beam tests of the Tevatron IPM show that the instrument can resolve turn-by-turn beam size oscillations in the order of $\sim 5 \%$ for single bunches at injection, and about the same sensitivity at top energy. The absolute accuracy, including systematic effects, is yet to be studied in detail. The initial tests were made at reduced electric and magnetic fields due to problems with sparking. These problems have been understood and corrected. Both detectors have been reinstalled during the spring shutdown, and further testing will be done when the machine comes up again in June, in order to fully explore the performance of the system.

\section{ACKNOWLEDGMENTS}

The Tevatron IPM project has been a collaborative effort involving people from all four major divisions at Fermilab (Accelerator Divison, Particle Physics Division, 
Computing Division and Technical Division). The authors would like to specially acknowledge the contributions of Mark Bowden, Alan Bross, Alex Chen, Bob Dysert, Scott McCormick, Hogan Nguyen, Claudio Rivetta, Terry Anderson, Hank Glass, Dave Harding, Bob Hively, Vladimir Kashikin, Dale Miller, Zhijing Tang, Jim Volk, Tom Zimmermann, and the Tev Techs.

\section{REFERENCES}

1. T. Zimmerman and M. Sarraj, A Second Generation Charge Integrator and Encoder ASIC, IEEE NS V433 (June 1996).

2. P. Moreira et al, "A Radiation Tolerant Gigabit Serializer for the LHC Data Transmission" in Workshop on Electronics for LHC Experiments, Stockholm, Sweden , 2001

3. A. Jansson et al., "An Ionization Monitor for the Tevatron" in IEEE Particle Accelerator Conferenc,. Knoxville TN, 2005 